7. Lee, HW et al 1998 Nature $392: 569-574$ 8. Kang. MK et al 1998. Cell Growth Diff 9:85-95. 9. Martin, $K$. et al. 1998. Exp. Cell Res. 240:In press. 10. Chiu, C.P. and Harley, C.B. 1997. Proc. Soc. Exp. Blol. Med. 214:99-106.

Harry Rubin replies: There are 30 references in the first few pages of my review' that show in various ways that cells are damaged when they are transferred from the organism to cell culture. The most telling cvidence against a genetically fixed replicative lifespan of human fibroblasts in vivo is that only a small percentage of cells survive the explantation and they die off at an ever-increasing rate as they are serially passaged ${ }^{2-4}$. The Hayflick limit refers, not to the original cell population, but to the last surviving clone.

Several of the most experienced and respected workers on cell senescence reject its relationship to normal aging ${ }^{5,5}$. MacieiraCoelho states, ". . . the term senescence is a misnomer that should only be used in an operational sense." Martin, author of the most authoritative and frequently cited paper on the inverse relation between the age of human fibroblast donors and replicative lifespan in culture? now reveals that the relation was found only in explants from cadavers but not in biopsies from living persons ${ }^{5}$.

There is no solid evidence for a continuous reduction in telomere length with the age of human donors. The reference usually cited shows no length reduction beyond the age of $20^{8}$. The recent paper coauthored by Blackburn, the preeminent authority on telomeres, finds no length reduction in leukocytes for 20 years beyond the age of four?.

The rationalization by Farragher et al. for the lack of effect on lifespan or cancer of telomerase knockout of mice ${ }^{10}$, i.e., that mice have longer telomeres than humans, does not hold water since mouse telomeres have large interchromosomal variations, with some as short as those of humans". All that the telomerase transfection experiments show ${ }^{12,13}$ is that a tumor virus construct aided by an overexpressed telomerase may carry out the first step of neoplastic transformation, namely immortalization, which is certainly in harmony with what we already knew about retrovirus infection.

As the idea of a fixed replicative lifespan does not stand up to critical scrutiny, the claim of telomere length as a division counter is insubstantial at best. This most emphatically does not mean that replicating (and nonreplicating) cells do not change with age, since they most certainly do. Nor does it mean that all the work on cell senescence is in vain, since it does show what cells do under severe continuing stress, i.e., exhibit damage and ultimately die. But it is simply a logical fallacy to interpret this as a genetically fixed replicative lifespan.
Depariment of Molecular \& Cell Biology University of California, Berkeley (hrubin@uclink2.berkeley.edu)

1. Rubin, H. 1997. Mech. Aging Dev. 98:1-35.

2. Merz, G.S., Jr. and Ross, J.D. 1973. J. Cell Physiol 82:75-80.

3. Smith, J.R. and Hayfllck, L. 1974. J. Cell Biol. 62:48-53

4. Rabinovitch, P.S. 1983. Proc. Nati. Acad. Sci. USA 80:2951-2955.

5. Gristofalo, V.J. et al. 1998. Proc. Natl. Acad. Sci. USA. In press.

6. Macieira-Coelho, A. 1998. Mech. Aging Dev. 103:105-109.

7. Martin, G.M. et al. 1970. Lab. Invest. 23:86-92.

8. Allsopp, R.C. et al. 1992. Proc. Natl. Acad. Sci. USA 89:10114-10118.

Frenck, R.W. et al. 1998. Proc. Natl. Acad. Sci. USA 95:5607-5610.

10. Blasco, M.A. et al. 1997. Cell 91:25-34.

11. Zijimans, J.M. et al. 1997. Proc. Natl. Acad. Sci. USA 94:7423-7428.

12. Bodnar, A.G. өt al. 1998. Science 279:349-352

13. Vaziri, H. and Benchimol, S. 1998. Current Biol. 8:279-282.

\section{RLU and studies using the luciferase reporter gene}

To the editor:

The use of gene therapy in the treatment of human diseases implies the use of a wide variety of techniques to introduce foreign genes into eukaryotic cells. Comparisons between the different transfection methods are necessary to assess their relative efficiency. Owing to its sensitivity, speed, and convenience, the firefly luciferase assay is commonly used for the quantitative reporting of genetic activity. In this assay, luciferin and $\mathrm{Mg}^{2+} /$ ATP are added to cellular or tissue extracts, and the light intensity is measured using a luminometer.

However, comparison of different transfection methods is compromised by high heterogeneity in the representation of the results. Some authors express luciferase signal in terms of relative light units (RLU)/mg of proteins ${ }^{1}$, others in RLU/number of cells ${ }^{2}$, or by RLU/transfected organ'. RLU, as suggested by its abbreviation, is a relative unit depending on the luminometers (each of them give their own relative values), and on the sample or on the volume of the luciferase substrate used. Moreover, even if some authors give the relationship between the found RLU number and the amount of purified luciferase, comparison is not possible according to the source of purified protein, its specific activity, and the reasons mentioned above.

To remedy this problem, a common representation should be adopted. For example, it would be more biochemically relevant to express the results in enzymatic activity of luciferase corresponding to the amount of enzyme causing transformation of 1 micromol of substrate per minute.

Several conditions are required to achieve successful comparison of results. First, results should be only compared when the enzymatic reaction between cellular luciferase and substrate occurs at maximal speed $\left(V_{\text {max }}\right)$, i.e., always when it uses an excess of substrate, which is generally the case. Second, authors should give the calibration curve between the signal they obtain with their luminometer under standardized conditions and the number of luciferase units used to perform their calibration.

This last point requires that providers of purified luciferase indicate the specific activity of the product (corresponding to the ratio of units of activity/mg of enzyme) instead of the weight of luciferase and of the correspondence with RLU. It is thus necessary to define a standard. We propose as a standard unit allowing comparison among all the published results the catalytic activity measured/unit of protein extract.

Jean-Michel Vicat, David Blum, Didier Wion, Alim-Louis Benabid, and François Berger INSERM U318, Preclinical Neurobiology CHU Michallon Pavillon B BP217X 38043 Grenoble Cedex S, France

1. Boussif, O. et al. 1995. Proc. Nat. Acad. Sci. USA
92:7297-7301.
2. Fender, P. et al. 1997. Nature Biotechnology 15:52-56.
3. Abdaliah, B. et al. 1996. Hum. Gene Ther. 7:1947-1954

\section{Errata}

In "Viral sequences enable efficient and tissue-specific expression of transgenes in Xenopus" (Nature Biotechnology 16:253-257, March 1998), the plasmid p2L contains one ITR from the left end, and one ITR from the right end of wild-type $A A V$, rather than two copies of the right $A A V$ as stated in the published article. The authors apologize for this error.

In the April editorial, "A shot in the arm for DNA vaccines," the crops rice, maize, and wheat were mistakenly referred to as dicotyledenous plants-they are monocots - and tobacco and soy were referred to as monocotyledenous-they are dicots.

The article "International patent filing" (Nature Biotechnology 16:479-480, May 1998) incorrectly identified London as the location of the European Patent Office (EPO). The EPO is in Munich, Germany.

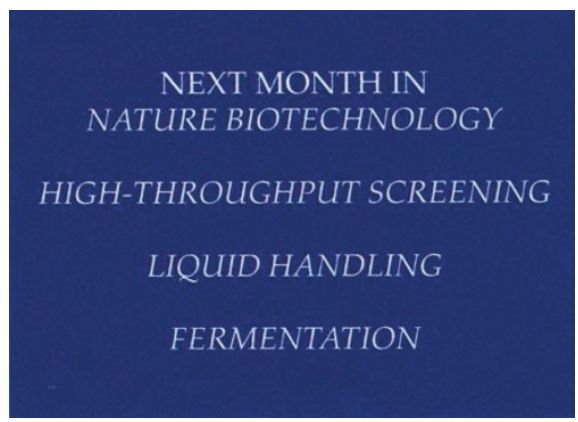

